Lerbinger - Zins- und Währungsswaps 
Dr. Paul Lerbinger

\section{Zins- und Währungsswaps}

Neue Instrumente im Finanzmanagement von Unternehmen und Banken 
CIP-Kurztitelaufnahme der Deutschen Bibliothek

\section{Lerbinger, Paul:}

Zins- und Währungsswaps: neue Instrumente im Finanzmanagement von Unternehmen u. Banken / Paul Lerbinger. - Wiesbaden: Gabler, 1988

(Die Bankgeschäfte)

Der Gabler-Verlag ist ein Unternehmen der Verlagsgruppe Bertelsmann

(c) Betriebswirtschaftlicher Verlag Dr. Th. Gabler GmbH, Wiesbaden 1988

Lektorat: Karlheinz Müssig

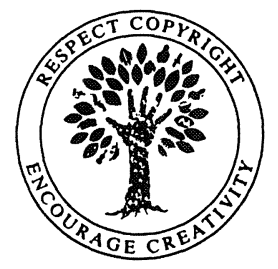

Das Werk einschließlich aller seiner Teile ist urheberrechtlich geschützt. Jede Verwertung außerhalb der engen Grenzen des Umeberrechtsgesetzes ist ohne $\mathrm{Zu}$ stimmung des Verlages unzulässig und strafbar. Das gilt insbesondere für Vervielfältigungen, Übersetzungen, Mikroverfilmungen und die Einspeicherung und Verarbeitung in elektronischen Systemen.

Satz: SATZPUNKT Ursula Ewert, Braunschweig

Buchbinder: Osswald \& Co., Neustadt/Weinstr. 
Inhalt

\section{Vorwort}

I. Grundlagen von Zins- und Währungsswaps

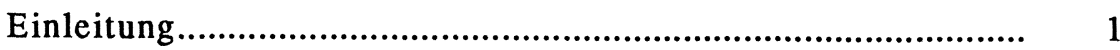

1. Frühe Form: Der Parallelkredit...................................................... 2

2. Entwicklung der Swaps................................................................. 3

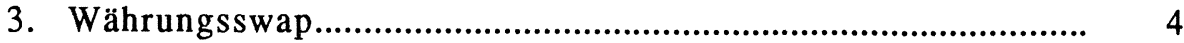

A Grundkonzeption .................................................................. 4

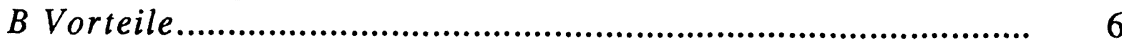

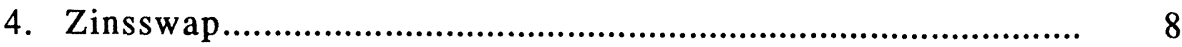

A Grundkonzeption ........................................................................ 8

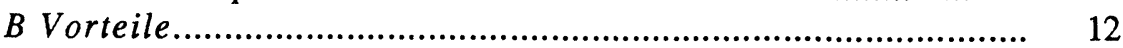

5. Zahlungsregelungen bei Swaps...................................................... 12

A Zahlungsstruktur mit individuellem Spot-Tausch.............................. 14

B Kompensierte Zahlungsstruktur..................................................... 14

6. Charakteristika der Swap-Märkte..................................................... 17

7. Einsatzmöglichkeiten von Swaps..................................................... 18

8. Rolle der Banken....................................................................... 20

II. Finanzmathematische Berechnungen

1. Berechnung der all-in Kosten bei der Begebung einer Anleihe.................. 22

2. Barwert- und Internal Rate of Return (IRR)-Analyse.............................. 27

3. Umwandlung von Renditen......................................................... 29

4. Spezielle Anwendungsbeispiele...................................................... 31

\section{III.Strukturen von Swaps}

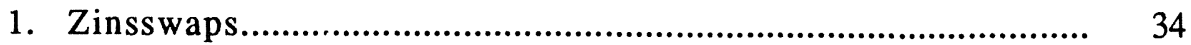

A Fixer-Variabler Zinsswap....................................................... 35

B Variabler-Variabler Zinsswap................................................... 39

2. Währungsswaps......................................................................... 41

A Fixer-Fixer Währungsswap..................................................... 43

B Fixer-Variabler Währungsswap................................................... 49

C Variabler-Variabler Währungsswap............................................... 51 


\section{IV.Innovationen bei Swaps}

1. Produktbezogene Innovationen............................................................... 53

A Forward-Swap ........................................................................... 53

B Amortisationsswap...................................................................... 55

C Aufbauender Swap................................................................... 61

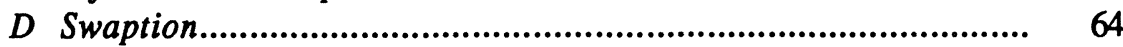

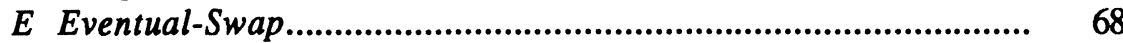

2. Neue Anwendungfelder................................................................... 69

A Internationale Schuldenkrise und Swaps........................................... 71

a) „Schulden-gegen-Beteiligung"-Swap................................................... 71

b) Asset-Swaps mit Staatsschulden..................................................... 74

B Waren-indexierte Swaps.................................................................. 81

3. Innovative Transaktionsstrukturen................................................... 85

A Internationale Aktienoptions-Anleihe ............................................... 85

B Mehrparteien-Swap .............................................................. 86

\section{Risikomanagement von Swaps}

1. Risikoidentifikation.................................................................... 90

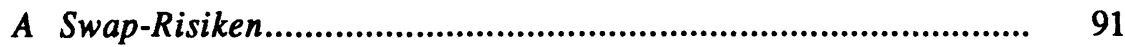

B Risikostruktur......................................................................... 92

2. Risikoschätzung............................................................................. 94

A Risikopotentialanalyse anhand von Beispielen................................... 95

a) Fixer-Variabler Zinsswap.......................................................... 95

b) Variabler-Variabler Zinsswap...................................................... 98

c) Fixer-Fixer Währungsswap.................................................... 99

d) Fixer-Variabler Währungsswap.............................................. 103

e) Variabler-Variabler Wăhrungsswap............................................... 105

B Ansatz zur Bestimmung des potentiellen Verlustrisikos....................... 106

3. Risikopolitische Maßnahmen..................................................... 108

A Risikovorbeugende Maßnahmen.................................................. 109

a) Bonitätsprüfung................................................................... 109

b) Sicherung durch Pfandrechte................................................. 110

B Risikoreduzierende Maßnahmen...................................................... 111

\section{VI.Swap-Technik}

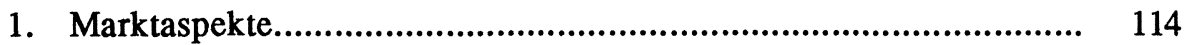

A Swap-Notierungen und Swap-Spreads............................................. 114

$B$ Währungsanleihen und Swaps................................................. 117

C Timing bei Währungsswaps.......................................................... 119 
2. Swap-Management................................................................... 125

A Umkehr-Swap ........................................................................ 125

B Asset-Swap........................................................................... 128

a) Aktives Zinsmanagement................................................... 128

b) Renditeverbesserung (Synthetische Papiere)................................. 130

C Zinsswap versus Cap........................................................... 138

3. Swap-Dokumentation............................................................. 142

A Vertrag.................................................................................... 142

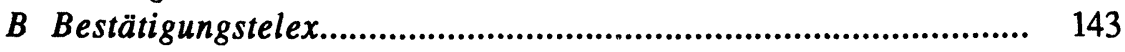

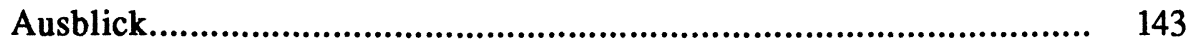

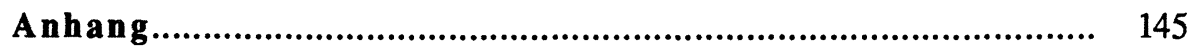

Standardisiertes Bestätigungstelex..................................................... 145

Swap-Indikation von Morgan Guaranty.................................................... 147

Libor-Sătze von Reuter................................................................... 148

Standardvertrag der International Swaps Dealers Association, Inc..................... 149 


\section{Vorwort}

Die meisten Erfindungen in unserem technischen Zeitalter, aber auch Neuerungen (Innovationen), kommen aus der Werkstatt des Wissenschaftlers, Technikers, Chemikers oder welches Spezialisten auch immer. Sie werden sozusagen auf dem Reißbrett gemacht, durchlaufen den komplizierten Weg der internen Prüfung, der Meldung beim Patentamt und werden dann als neues Produkt dem Markt angeboten.

Das Finanzgeschäft, insbesondere das internationale, geht hier einen ganz anderen Weg. Echte Innovationen werden nicht von heute auf morgen erfunden, sondern sie entwickeln sich aus dem täglichen Geschäft, das nicht nur einem offenen, sich steigernden Wettbewerb ausgesetzt ist, sondern sich auch gesetzlichen, aufsichtsrechtlichen und wirtschaftspolitischen Auflagen anpassen muß.

Der Zins- und Währungsswap ist eine solche Innovation, die der Praktiker entwickelt, verfeinert und ständig verbessert hat. Der Grundgedanke ist die Arbitrage von Vorteilen, eine dem Homo sapiens - zumal wenn er im Finanzwesen geschult ist - nicht unübliche Handlung. Dies wird wohl auch einer der wesentlichen Gründe dafür sein, daß diese Innovation, die - wie der Autor ausführt - Anfang der 80er Jahre ihren Siegeszug begann, auf soviel Anwender und Nachahmer gestoßen ist. Mit Recht mißt daher der Autor dieser Finanzierungsart eine Drehscheibenfunktion zu, womit sie im nationalen und internationalen Finanzierungsgeschäft zum Ausgangspunkt vieler Finanzierungsvorhaben wurde und bleiben dürfte.

Waren es zunächst die unmittelbar am internationalen Finanzgeschehen beteiligten Banken, die sich zur Verbesserung ihrer Refinanzierungskonditionen dieses Instrumentes bedienten, - und dies war ein direkter Ausfluß der Schuldenkrise, die Anfang 1982 die intemationalen Kredit- und Kapitalmärkte zu erschüttem begann - so erwuchs die Anhängerschar in den darauf folgenden Jahren aus dem Unternehmensbereich.

In vielen Seminaren und Tagungen ist der Zins- und Währungsswap und seine verschiedenen Fortentwicklungen auch dem deutschen Finanzmanager nahegebracht worden. Es ist das Verdienst des Autors, erstmalig in der deutschsprachigen Fachliteratur eine detaillierte Abhandlung über eine der bedeutendsten Finanzinnovationen dieses Jahrzehnts zusammengefaßt zu haben. Bereichert wird dieses Werk durch die persönlichen Erfahrungen mit diesem Instrument, die der Autor in seine Darstellungen mit einfließen läßt. Damit wird diese nicht unkomplizierte Lektüre sehr lesenswert.

Frankfurt, den 29. März 1988

Dr. Rolf Levedag
Mitglied des Vorstandes
Schweizerischer Bankverein
(Deutschland) AG 